\title{
La prédication " nostrologique ». Quelques réflexions sur la nature du politique
}

Laurence Kaufmann

\section{OpenEdition}

\section{Journals}

Édition électronique

URL : http://journals.openedition.org/ress/594

DOI : 10.4000/ress.594

ISSN : $1663-4446$

Éditeur

Librairie Droz

Édition imprimée

Date de publication : 1 août 2002

Pagination : 283-308

ISBN : 2-600-00806-3

ISSN : 0048-8046

Référence électronique

Laurence Kaufmann, « La prédication «nostrologique ». Quelques réflexions sur la nature du politique », Revue européenne des sciences sociales [En ligne], XL-124 | 2002, mis en ligne le 01 décembre 2009, consulté le 20 avril 2019. URL : http://journals.openedition.org/ress/594 ; DOI : $10.4000 /$ ress.594 


\title{
Laurence KAUFMANN
}

\section{LA PRÉDICATION «NOSTROLOGIQUE» QUELQUES RÉFLEXIONS SUR LA NATURE DU POLITIQUE}

\begin{abstract}
RÉSUMÉ
Le politique est généralement conçu comme l'ensemble organisé des procédures nécessaires à l'élimination ou à la résolution des conflits qui opposent les intérêts particuliers et l'intérêt général, les individus et la cité. L'enquête ontologique, conformément au cadre analytique qui est le sien, fait d'une telle assomption son objet d'investigation principale. Elle se penche sur les processus formels qui transforment des particuliers hétérogènes en un Nous relativement homogène et des élaborations linguistiques en des institutions contraignantes. Le but de cet article consiste ainsi à montrer, étape par étape, que la double conversion des faits sociaux en «choses» publiques et des individus en agent collectif constitue l'essence même du politique.
\end{abstract}

\section{INTRODUCTION}

Placer le politique sous l'égide d'un questionnement ontologique peut sembler à plus d'un titre paradoxal - sinon déplacé. En effet, l'ontologie est traditionnellement définie comme l'étude des entités basiques qui existent dans le monde réel, au-delà des apparences illusoires et des connaissances erronées qui rendent provisoirement leur accès impraticable ${ }^{1}$. L'ontologie interroge la «Réalité » des choses dont l'existence est indépendante des savoirs et des discours contingents que les individus sont susceptibles d'entretenir à leur égard. De prime abord, le questionnement ontologique, qui semble se préoccuper exclusivement de «ce qui existe» du côté des objets, ne concerne donc guère les sciences sociales et politiques, dont le champ d'investigation est constitué par les savoirs, les discours et les pratiques qui prédominent $d u$ côté des sujets de connaissance et d'action. Pourtant, une réflexion plus approfondie montre clairement que la dérobade ontologique qu'arborent les sciences sociales et politiques est largement injustifiée. Les théories qu'elles privilégient mettent en effet nécessairement en œuvre des engagements ontologiques implicites qui plaident en faveur de l'existence objective d'un certain nombre d'entités de base - que ce soient les structures institutionnelles, les classes socio-économiques, les pratiques réglées, les représentations collectives, les acteurs stratégiques ou les systèmes de bonnes raisons ${ }^{2}$.

P. van Inwagen «The Nature of Metaphysics» in S. Laurence \& C. Macdonald (éd.) Contemporary Readings in the Foundations of Metaphysics, Oxford/Massachusetts, Blackwell, 1998, pp. 11-21.

2 Sur cette notion essentielle d'engagement ontologique, voir W.V.O. Quine Le mot et la chose, Paris, Flammarion, 1977, ainsi que W.V.O. Quine «On What There Is » in S. Laurence \& C. Macdonald (ed.) op. cit., pp. 32-45. 
Une démarche ontologique explicite récuse précisément l'insertion inquestionnée et a priori de ces «particules élémentaires » dans des systèmes explicatifs sinon des chaînons causaux. Conformément au principe de parcimonie et à l'économie conceptuelle préconisés par la méthode analytique, elle tente d'éviter l'inflation des notions superflues. En conjurant ainsi la multiplication inutile des concepts et a fortiori des ordres de réalité pour expliquer des phénomènes dont des entités d'ores et déjà reconnues suffisent à rendre compte, une telle perspective se donne les moyens de spécifier l'ontologie quelque peu saugrenue des faits sociaux et politiques. C'est du moins l'enjeu de cet article, qui vise précisément à montrer qu'une démarche ontologique peut aider à discriminer, étape par étape, les propriétés formelles des phénomènes politiques. Bien entendu, l'enquête ontologique a le défaut de ses avantages: en cherchant à cerner les propriétés invariantes du politique, elle laisse par définition de côté la particularité et la complexité de ses actualisations socio-historiques. Cela étant, ces mêmes actualisations empiriques ne pourraient être rendues sans le recours, implicite ou explicite, à un cadrage théorique préalable qui les rende préhensibles. C'est précisément ce cadrage préalable que l'investigation ontologique prend pour objet, puisqu'elle vise à expliciter ce qui fait l'ordinaire tacite du sociologue et du politologue. Elle vise également à dépasser l'hermétisme des écoles de pensée en déployant un terrain de discussion, sinon d'entente, quant aux distinctions conceptuelles de base qui sont susceptibles de les rassembler. L'enquête ontologique, dans la mesure où elle délaisse la chair historique des faits sociaux pour le squelette formel de leur structure, constitue plus un prélude qu'une fin en soi. Mais un tel prélude s'avère indispensable lorsque l'on veut éviter que les scissions théoriques et la profusion des apports empiriques n'engendrent un monde social surpeuplé, polymorphe, inconsistant, bref, ingérable. C'est du moins à un tel pari que s'attèlent les pages qui suivent.

\section{LES ARCANES DU POLITIQUE}

Les disciplines concernées par la notion même de politique sont désormais familières avec la fameuse distinction qu'ont préconisé, chacun à leur manière, Claude Lefort et Cornelius Castoriadis ${ }^{3}$. D'un côté, le politique renvoie à un invariant anthropologique: toute collectivité doit fixer de son propre chef les repères du réel et de l'imaginaire, du vrai et du faux, du bien et du mal, du possible et de l'impossible ou encore du juste et de l'injuste ${ }^{4}$. De l'autre côté, la politique est la forme explicite prise par la fixation de ces repères dans une société démocratique, idéalement débarrassée des fondements substantiels qui rendaient les institutions traditionnelles hermétiques à l'action humaine (les totems, les ancêtres, Dieu ou la nature). L'émergence de la politique à un moment donné de l'histoire scelle ainsi une intellection nominaliste des institutions: loin d'être des créations aveugles dont les origines se perdent dans les us et coutumes du passé, elles sont

\footnotetext{
C. Lefort, Les formes de l'histoire. Essais d'anthropologie politique, Paris, Gallimard, 1978.

C. Castoriadis, Domaines de l' homme. Les carrefours du labyrinthe II, Paris, Seuil, 1986.

4 Lefort, op. cit.
} 
des constructions artificielles soumises au bon vouloir lucide des citoyens, désormais crédités du pouvoir de créer les règles qui régissent leur coexistence. Dans ce cadre 'autonomique', l'acte d'origine de l'institution ne peut que s'inscrire dans l'espace public de justification et de manipulation que déploient «les hommesdésormais-entre-eux ». La collectivité, ainsi consacrée comme l'ultime instance de référence et de légitimation, se voit officiellement dotée du pouvoir fondamentalement politique que constitue la hiérarchisation des pratiques et des valeurs concernées par l'intérêt général.

Dans la mesure où l'avènement de la politique annonce, dans l'espace ouvert de la citoyenneté, la mise en question publique des orientations collectives, la description et l'interprétation des rapports sociaux représentent un enjeu plus fondamental que jamais. En effet, une fois homologués la relativité des significations et le statut conventionnel des règles, seule la justification politique est à même d'assurer «l'auto-intelligibilité » d'une société qui n'a plus rien d'autre pour se soutenir, semble-t-il, que l'ouvrage incessant de «l'énonciation de la parole» ${ }^{5}$. La parole publique étant désormais chargée de lester les repérages collectifs du poids nécessaire au maintien de la cohésion sociale, elle devient l'enjeu vital d'une compétition discursive sans merci entre les aspirants au pouvoir. Le politique, lorsqu'il devient l'objet explicite de la délibération publique au XVIII e siècle, n'est donc pas uniquement synonyme d'émancipation, car il est constamment menacé par le jeu global, simplifiant et schématique, de l'idéologie. Pour une collectivité qui a pris acte, politiquement et juridiquement, de son auto-constitution, l'idéologie représente le moyen méta-discursif de restreindre la circulation du sens en lui imposant les bornes d'un savoir prétendument objectif sur le social.

De cette brève synthèse, nous pouvons retenir trois éléments clés qui reviendront sous différents aspects tout au long de notre parcours. D'une part, le principe de l'auto-détermination des régimes démocratiques met en exergue le constat métaphysique selon lequel la société est une construction artificielle. Un tel nominalisme social et politique confère aux membres de la communauté le pouvoir de réaliser, au sens littéral du terme, les objets sociaux dont ils reconnaissent collectivement l'existence ${ }^{6}$. D'autre part, les faits sociaux étant conçus comme la création originaire de la collectivité, c'est à la politique en tant que telle que revient la tâche, normativement parlant, de faire «tenir ensemble» les deux versants du

C. Lefort, «Penser la révolution dans la Révolution française», Annales, n², 1980, p. 349 et sq.

6 Il nous faut préciser ici que le nominalisme est une dénomination qui se suffit rarement à ellemême; il se décline la plupart du temps accompagné d'un qualificatif qui précise son acception, tels que nominalisme scientifique, nominalisme empiriste, nominalisme logique, nominalisme social ou politique, etc. C'est à ce dernier que nous faisons allusion: il renvoie à l'idée que les institutions ne sont pas a priori antérieures ou supérieures aux individus singuliers qui les constituent. Tout en prenant un tel cadre comme notre point de départ, notre but n'est aucunement d'arriver à la conclusion que les institutions sociales et politiques ne seraient que de simples noms ou des manières commodes de parler. Il est plutôt de montrer pourquoi les entités sociales et politiques, bien que nominales, bénéficient bel et bien d'une forme de réalité irréductible. Sur le nominalisme et ses multiples versions, voir Jean Largeault, Enquête sur le nominalisme, Paris/Louvain, Nauwelaerts, 1971, ainsi que C. Panaccio, Les mots, les concepts et les choses. La sémantique de Guillaume d'Occam et le nominalisme aujourd' hui, Montréal/Paris, Bellarmin/Vrin, 1991. 
monde social, à savoir l'individu et l'institution. Enfin, l'idéologie, en prétendant énoncer des vérités ultimes sur le social, tend à distordre la nature conflictuelle et délibérative de la politique en lui imposant le mode de légitimation objectiviste propre au savoir scientifique. Or, à mon sens, la configuration qui relie nominalisme, politique démocratique et idéologie gagne à être retraduite dans un format analytique qui évite la consécration, paradoxalement «substantialisante», de «la société » comme étant le nouveau sujet agentif de l'histoire. En effet, plutôt que de partir de l'entité quelque peu énigmatique que constituerait la société et de mettre en œuvre les instruments «magmatiques » qui lui sont corrélatifs, mieux vaut tenter de l'appréhender sous les auspices des micro-procédures qui travaillent continuellement à sa production et à sa rigidification. En délaissant la conception de la société accomplie pour les modalités de son accomplissement, une telle démarche avalise le constat nominaliste qui a permis aux communautés traditionnelles de rompre le secret public qui avait été, jusqu'alors, soigneusement passé sous silence: la «Société» n'est qu'une société dont la reconduction dépend du bon vouloir de ses membres.

L'approche que nous préconisons ici, en privilégiant le versant «en amont» de la politique interne de cette reconduction plutôt que sa résultante «en aval», se donne les moyens de cerner la teneur étrange du pouvoir proprement constituant que détient le Nous de société. Ce pouvoir, c'est celui de transformer les objets intentionnels communs qui émergent à la croisée des esprits individuels en des entités bel et bien réelles, dotées de la consistance et de la résistance généralement réservées aux espèces dites «naturelles » ${ }^{7}$. Ce pouvoir hors du commun, et pourtant ordinaire, qui fait de la collectivité le dieu social au principe de l'être intentionnel des institutions et des pratiques mérite que l'on s'y attarde. Car il renvoie au procès politique par excellence que constitue la détermination et la constitution collective de la «chose publique». D'un point de vue ontologique, en effet, la res publica est une armature relativement figée qui résulte de la montée en généralité et de la sédimentation des volontés et des actions individuelles. Le politique, qu'il recoure à la logique transcendante des êtres supra-naturels ou à la logique immanente de la politique, peut ainsi être appréhendé comme un travail au long cours. Ce travail, c'est celui qui permet de fabriquer du général et de l'objectif à partir du matériau de base que sont les subjectivités individuelles.

Or, le chemin fondamentalement politique, qui mène ainsi de l'insubordination de la liberté individuelle et du caprice de l'opinion particulière à l'institution d'un monde commun, s'avère largement énigmatique. Il est en tous les cas énig-

Le terme «intentionnel» renvoie à la propriété générale des états mentaux, tels que croyance, désir, intention, motif, volonté, etc. La notion d'intentionnalité, très utilisée en philosophie analytique, est plus riche que la notion quelque peu statique de «mental» car elle exprime la relation fondamentale de l'individu au monde qui l'entoure et constitue donc la marque distinctive du monde mental et social par rapport au monde physique. La notion d'intentionnalité désigne la capacité qu'a un être humain de renvoyer à quelque chose qui se situe en dehors de son esprit, la possibilité de penser ou de parler à propos d'un objet ou encore de se représenter «dans la tête» un référent même en l'absence de celui-ci. La pensée d'un individu est donc nécessairement intentionnelle, car elle se réfère à un état du monde, réel ou imaginaire, qui attire son attention et devient par là même son «objet intentionnel». Sur cette notion d'intentionnalité, voir notamment J. Searle, L'Intentionalité. Essai de philosophie des états mentaux, Paris, Minuit, 1985 et R. Chisholm, «Intentionality», in The Encyclopedia of Philosophia, New York, Macmillan, 1972. 
matique si l'on évite, ne fût-ce que provisoirement, de dissoudre le problème de la conversion de l'individuel au général dans le postulat d'un «toujours déjà-là » de l'institution ${ }^{8}$. C'est d'ailleurs cette même énigme qui hante les tenants lucides de l'auto-détermination démocratique: dans un monde où les individus sont officiellement promus comme étant les seuls membres légitimes du corps social, comment donc reconstruire une totalité sociale à partir des atomes que sont les personnes privées? L'option sociologique traditionnelle se contente généralement de rappeler que cette question est une pure construction de l'esprit: en soustrayant de son équation politique la pesanteur de la tradition et des usages établis, qui font par définition de tout individu «un personnage public», elle ne ferait que succomber aux velléités créationnistes des irréalistes, des intellectuels et des révolutionnaires. A l'encontre d'un tel diagnostic démystificateur, notre option ontologique se propose précisément de revenir naïvement, conformément en cela à l'étonnement philosophique, sur la charade redoutable auquel se heurte tout système politique: mon premier est un, mon deuxième est un, et mon tout est public. Une telle charade, même si elle repose la vieille question, pratique et théorique, de «l'institution de l'institution», n'aboutit pas nécessairement à une version conventionnaliste, individualiste ou contractualiste, de la vie en commun. C'est ce que nous allons tenter de montrer en nous centrant sur la transmutation mystérieuse des actions et des références intentionnelles, théoriquement dépendantes des esprits qui les mettent en œuvre, en des «objets» publics partiellement autonomes. Dans la mesure où cette transmutation saugrenue est une bizarrerie épistémologique qui nous amène au cœur même du politique, c'est à son explicitation qu'il nous faut maintenant nous intéresser.

\section{L'ÉTRANGE ONTOLOGIE DES FAITS SOCIAUX}

La propriété ontologique la plus fondamentale des faits sociaux et politiques se retrouve dans la division générique, d'origine aristotélicienne, entre la physique des particuliers concrets et naturels, dont la réalité est directement ou indirectement accessible aux sens, et la méta-physique des entités surnaturelles et des abstractions artificielles, qui ne doivent leur existence qu'au travail de l'esprit et du langage. Bien entendu, la valorisation des réalités qui tombent, littéralement parlant, sous les sens (toucher, vue, ouïe, goût ou odorat) a bien souvent relégué la métaphysique au rang inférieur des êtres immatériels et invisibles, des jeux de mots fictifs et des projections imaginaires. Mais les fâcheuses conséquences d'une telle division n'implique pas l'invalidation de son précepte de base, que résume fort bien Ian Hacking. Les entités physiques, tels le tigre, le quark ou l'eau, sont des «espèces indifférentes » aux discours dont elles font l'objet, leurs propriétés primitives, essentielles, autonomes et nécessaires (les rayures, l'acidité

A cet égard, notre démarche se distingue d'une conception holiste a priori, comme celle de Vincent Descombes: celle-ci, en insistant sur la matrice primordiale de pré-jugements et de pratiques que l'enfant a «avalé » au cours de son apprentissage, constitue essentiellement une théorie de la reconduction des institutions - une théorie qui, aussi intéressante soit-elle, ne peut guère rendre compte de la création des institutions ou du changement socio-historique. Cf V. Descombes, Les Institutions du sens, Paris, Minuit, 1996. 
ou les molécules H2O) étant hermétiques au traitement que les humains leur imposent. En revanche, les entités sociales et politiques, tels l'opinion publique, le pouvoir ou la maltraitance, sont des «espèces interactives» dont les propriétés hétéronomes et contingentes dérivent de «l'art», plus ou moins volontaire, des bricoleurs ou des ingénieurs que sont les agents ordinaires ${ }^{9}$. Autrement dit, contrairement aux espèces naturelles, «l'existence» des espèces sociales - qui prend plutôt la forme, pour les physicalistes, d'une inexistence-est artefactuelle, car elle est «faite» et «défaite» par des êtres humains socialement et historiquement situés ${ }^{10}$.

Comme le dit John Searle, les êtres sociaux et politiques se caractérisent donc par une ontologie subjective : leur existence dépend de leur reconnaissance, car ils sont ce qu'ils sont si et seulement si les gens pensent qu'ils sont ce qu'ils sont ${ }^{11}$. Ontologiquement parlant, l'existence d'un fait social relève de l'accord collectif des agents qui ont pu, grâce à la capacité de symbolisation que leur offre la mâ̂trise du langage, attribuer des significations à des faits bruts ou naturels a priori insignifiants ${ }^{12}$. Les faits sociaux sont les corrélats intellectuels et pratiques de la mise en commun des esprits individuels, ce qui fait que leur ontologie est une ontologie en Nous: ils s'effacent lorsqu'ils ne sont plus reconduits et actualisés par la collectivité qui lui prête momentanément existence. Ainsi, si plus personne n'est capable d'appréhender et de mettre en pratique des institutions données, que ces institutions soient considérées au sens générique d'une loi établie ou d'une habitude (la prière) ou au sens spécifique d'une organisation répondant à une

9 Cf. I. Hacking, The social construction of what?, Cambridge/London, Harvard University Press, 1999.

10 Notre démarche, en insistant sur la différence ontologique, s'oppose à de nombreuses formes de constructivisme qui tendent à rapatrier les sujets et les objets dans un même univers artificiel et, ce faisant, à étendre à l'infini la juridiction du langage et de l'esprit. Pour la fameuse «ontologie à géométrie variable » de Bruno Latour, par exemple, le monde est enveloppé dans des «faits » qui ne sont que les conséquences relativement stables des classifications, des instrumentations et des accords convenus par l'intermédiaire desquels nous les construisons: du coup, la seule «matière » des sciences serait celle des «manières de faire ». Cette démarche, par ailleurs fort cohérente, n'est toutefois pas sans soulever quelques embarras, tout au moins dans le cadre qui est le nôtre: en amalgamant faits sociaux et faits bruts dans une même chaîne de construction qui ne varie que par sa longueur et par le mode de lestage de ses maillons, elle annule purement et simplement l'enquête ontologique. Voir entre autres Bruno Latour, Petite réflexion sur le culte moderne des dieux faitiches, Paris, Ed. des Empêcheurs de tourner en rond, 1996.

11 J. Searle, «Intentionalistic Explanations in the Social Sciences», Philosophy of the Social Sciences, vol. 21, 1991, n³, pp. 331-344.

12 Nous reviendrons sur la nature de cet accord. Précisons toutefois d'emblée que la conception de Searle, aussi intéressante et commode soit-elle, tend à privilégier une conception volontariste et instrumentale des institutions qui ne sera aucunement la nôtre. Son exemple favori de l'institution est le concert de musique: ce dernier est le produit des volontés individuelles, qui unissent provisoirement leurs contributions et leurs capacités pour réaliser un but commun, en l'occurrence un concert. Tout en reprenant à notre compte l'essentiel de son argument, c'est-à-dire la dimension ontologique, nous défendrons une conception tout à fait différente du Nous au principe des institutions. On le verra, le Nous en question, loin d'être suspendu à une alliance volontaire et égalitaire, se caractérise par une structure interne hiérarchique et une autonomie d'action qui lui permettent «d'échapper» aux membres qui le composent. 
fonction publique (l'église), elles meurent définitivement ou disparaissent dans l'histoire lointaine des us et coutumes.

Dans la mesure où les faits sociaux et politiques n'existent que lorsqu'ils sont publiquement perçus et reconnus par une communauté, ils ne sont «que»des constructions durcies par l'usage, les évidences partagées et les dispositifs institutionnels. Et pourtant, en dépit de leur précarité ontologique, ils apparaissent bien souvent lestés du poids apparemment immuables des choses en soi, leur irréductibilité justifiant ainsi le réalisme social et sociologique dont ils font l'objet. Pour mieux saisir les arcanes de cette irréductibilité, il nous faut retracer le processus de base qui dote les créatures intentionnelles de l'inertie et de l'objectivité auxquelles seules les entités «indifférentes » auraient théoriquement droit. Ce processus est le procès de «factualisation» qui permet de transformer des chimères provisoirement communes en des choses, ou du moins en des entités de référence. Un tel procès, qui relie les agents ordinaires aux mêmes êtres intentionnels, renvoie à ce que Francis Jacques appelle un procès de «co-référenciation ${ }^{13}$. En effet, si tant est que l'on évite de poser a priori la primauté des faits institutionnels pour partir des esprits individuels, la création collective qui se conclut par l'instauration des «choses» publiques commence par une co-référenciation. Sous les auspices de ces procédures de co-référenciation, on le verra, le pouvoir performatif de l'intentionnalité collective à l'origine des institutions apparaît nettement moins mystérieux. Car le Nous qui précède et résulte tout à la fois des actions et des paroles concertées s'avère logiquement doté de la capacité de fixer et d'objectiver les «interréférences» de ses membres dans des objets intentionnels communs. C'est donc en analysant ces «interréférences» que nous pourrons mieux comprendre in fine comment les êtres étranges que sont les Nous collectifs peuvent présider, via les éléments ou plutôt les parties en Je qui les composent, à la détermination des existants sociaux et politiques.

\section{LE DIALOGUE RÉFÉRENTIEL}

Dans la perspective dialogique que propose Francis Jacques, le dialogue, loin de se contenter de présupposer un monde commun, est le moyen de sa continuelle auto-institutionnalisation par des individus qui parviennent à s'entendre sur ce qu'ils disent, à s'accorder sur ce dont ils parlent et à fixer ce à quoi ils font référence $^{14}$. Dans un tel cadre, la signification n'est aucunement suspendue aux fils mentaux des individus: déterminée «au point de jonction ou de friction entre ce

13 Sur ces processus de co-référenciation, voir F. Jacques, Dialogiques I. Recherches logiques sur le dialogue, Paris, PUF, 1979.

14 Précisons ici que l'approche dialogique de la communication que propose Jacques diffère nettement de la théorie de l'agir communicationnel de Jürgen Habermas qui met en scène «un jeu de langage idéal » censé être, par un étrange retournement, la condition de possibilité de tous les jeux de langage réels. Ce faisant, il occulte quelque peu la construction ordinaire des mondes à laquelle contribuent sans relâche les procédures du langage quotidien. Ce sont précisément ces procédures qui intéressent Jacques. Cf. F. Jacques, Dialogiques II. L'espace logique de l'interlocution, Paris, PUF, 1985, p. 375 et sq. 
que prononce le locuteur et ce que comprend l'auditeur», elle émerge de la transaction dialogique qui leur permet, en tant que $J e$ et $T u$, de désigner de concert un objet commun ${ }^{15}$. L'interlocuteur est donc toujours un «allocutaire», c'est-à-dire un colocuteur; de même, la relation de référence qui renvoie à un état de choses, réel ou fictif, est toujours une «co-référence». Afin d'établir cette co-référence, les allocutaires se basent sur le «contexte élargi» que forment les attentes et les croyances qu'ils s'attribuent mutuellement, ainsi que les faits publics que tous les membres de la communauté sont censés connaître. Au sein de ce contexte élargi, les partenaires de la communication ajustent progressivement «leurs mondes possibles» respectifs, c'est-à-dire «l'aire de possibilité» sémantique que déploie l'ensemble, actuel et virtuel, des présuppositions et des attitudes qu'ils entretiennent à l'égard du monde qui les entoure ${ }^{16}$. La mise en communicabilité des «mondes possibles», loin de se contenter d'un échange statique des états d'esprits, repose sur l'unité anticipée d'un consentement intersubjectif. La réalité sur laquelle porte l'accord dialogique est donc par définition transactionnelle: elle relie étroitement «le registre allocutif», qui exprime la dépendance des énonciations par rapport au $J e$ et $T u$ de leurs co-énonciateurs, et le « registre délocutif» qui marque, quant à lui, la désignation conjointe des objets d'un «tiers monde» qui a le statut impersonnel d'un Il ou d'un cela $^{17}$.

Bien entendu, la relation entre le registre intersubjectif de l'allocution et celui, objectivant, de la délocution, est bien différente quand la communication recourt, sur le mode de l'évidence, à des référents d'ores et déjà établis ou qu'elle déclenche, à l'inverse, une enquête conjointe quant à des entités encore indéterminées. Ainsi, certains dialogues sont essentiellement délocutifs, car ils portent sur des référents notoires et nécessairement partagés, que ce soit un fait public (le roi Louis XVI), une expérience commune (la promenade de l'autre jour), une perception convergente (cette rose) ou un concept ordinaire (un siège). Dans le cadre délocutif que déploient les phénomènes impersonnels dont l'existence fait d'emblée l'objet d'une reconnaissance publique, l'enquête dialogique s'avère superflue. La créativité et les tâtonnements conjoints sont désamorcés par l'autorité asymétrique d'un tiers hétéronome- une institution, un expert ou un porte-parole - qui imposent d'emblée l'identité des référents dont doivent tenir compte tous les membres de la communauté ${ }^{18}$. En revanche, lorsque le sens et/ou la référence d'un événement, d'un concept ou d'une situation suscitent un «questionnement conjoint», la communication prend la tournure essentiellement allocutive de ce que Jacques appelle le «dialogue référentiel».

15 F. Jacques, Dialogiques I. Recherches logiques sur le dialogue, Paris, PUF, 1979, p. 137. Voir aussi F. Jacques, Différence et subjectivité. Anthropologie d'un point de vue relationnel, Paris, Aubier, 1982.

6 Jacques (1979) p. 226.

17 Jacques (1979) p. 101

18 Jacques (1979) p. 162. A cet égard, le cadre épistémique de la recherche d'informations constitue un bon exemple: dans des questionnements tels que «qui est le roi de France au XVe siècle?», «quelle est la racine carrée de 198 ?» etc., le référent est déjà fixé par l'observation, la mémoire cumulative, la démonstration ou le calcul, ce qui rend l'enquête dialogue totalement superflue. 
Notre enquête ontologique visant à analyser la venue au monde social des phénomènes impersonnels que sont les concepts et les valeurs politiques, elle ne traite pas en tant que tel de l'ordre délocutif des faits institués et de l'autorité tierce qui y préside. Elle tente plutôt d'éclaircir ce tiers énigmatique et les faits abusivement délocutifs qu'il régit en se penchant sur les processus en amont de leur fabrication et de leur institutionnalisation. Or, les faits institués, bien avant de devenir les «choses » publiques que tout un chacun est tenu de connaître, ont commencé par être les intersections provisoires d'un dialogue référentiel plus ou moins élargi. En effet, le dialogue « référentiel» vise à spécifier, par approximations et ajustements successifs, un nouveau référent en partant des informations disponibles entre les interlocuteurs, ce qui fait que «ce que l'un répond à l'autre ne peut aucunement se conclure du discours d'un troisième ${ }^{19}$. Il concerne donc les idées, concepts ou faits qui n'ont pas encore été fixés par un argument d'autorité ou une démonstration empirique et qui ne peuvent que reposer, par conséquent, sur le jeu éminemment coopératif des partenaires. Dans ce jeu coopératif, les interlocuteurs procèdent à l'alignement de leurs croyances et connaissances respectives et fixent, au terme de leur enquête commune, un référent encore indéterminé. Le dialogue référentiel vise ainsi à statuer sur le sort des «individus possibles » qui ne sont pas encore des entités avalisées par l'expérience perceptive ou/et les croyances collectives, mais qui sont néanmoins jugés aptes à être candidats à la référence. Par exemple, les membres de la communauté scientifique, lorsqu'ils sont confrontés à une substance chimique encore inconnue, tentent de déterminer de conserve sa composition moléculaire (ie. son sens, par ex. H20) et de fixer les critères qui permettent d'identifier avec certitude les objets du monde (ie. ses référents, par ex. eau) auxquels la composition ainsi déterminée est susceptible de s'appliquer.

Le dialogue référentiel, et c'est ce qui est intéressant pour notre problématique, ne se contente pas de traiter et à terme d'éliminer les connaissances douteuses encore confinées dans le purgatoire épistémologique des sciences. Il intervient également et surtout lorsque l'enquête tente de donner forme à des aspirations nouvelles ou à des événements a priori incompréhensibles en élaborant des descriptions et des dénominations inédites. Ces innovations sémantiques, dont la création des institutions est nécessairement partie prenante, sont des entités intentionnelles qui ne font pas, du moins pas encore, l'objet d'un savoir délocutif qui se serait affranchi de ses origines allocutives. De telles entités n'ont pas la même réalité que les espèces «indifférentes » car leur existence potentielle et $a$ fortiori le recensement de leurs propriétés restent suspendus à une clause de croyance. Mais cette clause de croyance, on va le voir, a une caractéristique majeure qui modifie drastiquement le statut initialement précaire de cette dépendance: elle est commune. Grâce à cette dimension commune, des objets intentionnels peuvent s'autonomiser des allocutaires qui leur ont pourtant prêté existence et s'imposer à eux sous l'égide d'un Nous dont la dynamique leur échappe.

En effet, le Nous, qu'il renvoie aux interlocutions ordinaires qui jalonnent le cours de la vie quotidienne ou aux dialogues référentiels à grande échelle que déclenchent les crises politiques, met un terme à la dualité initiale des interlocuteurs en les intégrant dans un sujet collectif dont ils deviennent les porte-parole.

19 Jacques (1979) p. 195 
Les prises de position respectives des allocutaires s'effacent donc derrière le réseau interdiscursif de leurs énonciations croisées; elles font place à une posture finale en Nous qui atteste de la mise en communauté de la référence et justifie l'attribution d'une seule et même attitude au collectif ainsi constitué. La «communautarisation» en Nous des pronoms personnels qui structuraient la situation originaire de l'interlocution (le $J e$ et $T u$ ) manifeste ainsi l'instauration d'une situation nouvelle ${ }^{20}$. Car le Nous a posteriori de l'interlocution, une fois qu'il a endossé la croyance commune des allocutaires impliqués dans sa constitution, enclenche un processus dit de «rétro-référenciation», qui force ces mêmes allocutaires à se reconnaître et à s'individuer en tant que membres d'une totalité collective qui désormais les dépasse. Ce Nous bénéficie donc d'un statut logique et grammatical tout à fait particulier par rapport aux éléments qui le composent: à la différence du $J e$ et du Tu, rendus instables par l'échange mutuel de leurs positions de locuteur et d'interlocuteur, le Nous formé par la communauté de pensée et de parole des allocutaires est stable. Alors que «le $J e$ et $T u$ constituent de simples nœuds provisoires et différentiels d'élocution », le Nous peut tout à la fois appartenir en commun à tous et en propre à chacun, ce qui fait qu'il constitue le seul point fixe de l'espace interlocutif, le seul «invariant de la variation » pronominale $^{21}$. De plus, le Nous, en tant que résultante de l'agencement réciproque des mondes possibles des allocutaires, ne peut plus être redistribué au terme de la co-référenciation. Le dit étant désormais l'action d'un sujet collectif, il est progressivement libéré du dire propre à la situation particulière d'énonciation et peut être repris par n'importe quel locuteur potentiel ${ }^{22}$.

\section{L'AUTONOMISATION DU NOUS}

Au vu de ce qui précède, même le «couplage relationnel» apparemment anodin de deux sources énonciatives est apte à générer un système d'ordre supérieur qui bénéficie du statut «auto-transcendant» dont parle Jean-Pierre Dupuy: par un processus dynamique d'ajustements mutuels, l'interlocution s'auto-organise de telle manière que les phénomènes collectifs qu'elle engendre sont plus complexes que les agents qui les ont engendrés ${ }^{23}$. Cette étrange propriété permet de mieux comprendre comment des idées qui ont émergé sous des auspices allocutives peuvent changer de statut une fois consacrées comme les objets intentionnels d'un Nous - que celui-ci soit limité à deux personnes ou s'élargisse à l'ensemble d'une communauté. En effet, des notions socio-historiques au départ approximatives et mal définies, telles que «opinion publique», «nation», «absolutisme», «liberté », «citoyenneté », etc, sont parvenues à s'imposer comme des nouvelles entités de référence grâce à leur prise en charge par un Nous relativement stable.

20 Il est à noter ici que l'on parle de «communautarisation» et non de «pluralisation», car les individus, loin de rester des identités distinctes provisoirement associées, se retrouvent inextricablement imbriqués dans un Nous qui les subsume sous une identité commune.

21 Jacques (1979) p. 337

22 Jacques (1979) p. 257

23 J.-P. Dupuy, Introduction aux sciences sociales. Logique des phénomènes collectifs, Paris, Ed. Marketing, 1992. 
Bien évidemment, ces nouvelles dénominations, a priori déviantes par rapport aux usages institués qui sont d'ores et déjà parvenus à accéder à l'ordre délocutif des «choses» dont on parle, ne peuvent être consacrées collectivement sans un esprit de coopération. Les allocutaires potentiels que représentent tous les membres de la communauté doivent accepter d'avaliser la prétention de validité, originellement abusive, des énonciations déviantes en les reprenant à leur propre compte. Grâce à cet aval collectif, de purs êtres intentionnels se voient crédités d'une validité dont les privait leur décalage initial par rapport au cadre linguistique et conceptuel dominant. Lestés de l'autorité en Nous qui résulte des dialogues référentiels réussis, ils peuvent alors endosser les oripeaux objectifs des «objets notionnels» dont parle Daniel Dennett.

A la différence des objets réels, les «objets notionnels » sont les corrélats des croyances «linguistiquement infectées» que partagent les membres d'une communauté plus ou moins élargie ${ }^{24}$. Les objets notionnels ne bénéficient donc pas du même statut ontologique que les espèces «indifférentes», car leur existence est subordonnée à une dimension d'usage: celle de «l'usage impersonnel qui fait loi » et que nul n'est censé ignorer ${ }^{25}$. Cela étant, en vertu de leur statut public et impersonnel, ils sont dotés d'une consistance sémantique «interne» suffisamment récalcitrante pour pouvoir faire l'objet d'actes de référence identifiants qui contraignent, in fine, leurs usagers. En effet, l'identité des co-référents sociaux et politiques qui meublent le contexte élargi d'une communauté de langage et de pratique n'est pas soumise à des aléas psychologiques et situationnels. Bien qu'ils ne puissent être identifiés sans les «lunettes» intentionnelles qui leur sont corrélatives, ils n'oscillent pas au gré du temps ou de l'espace situés de leur instanciation. Dans le contexte des macro-relations intentionnelles et impersonnelles dont le Nous de société est le garant ultime, les objets notionnels répondent à des conditions de validité persistantes. Ainsi, des référents tels que «l'opinion publique» peuvent être remplacés par des termes socialement équivalents qui préservent leur validité, en l'occurrence «la somme des opinions individuelles». A la différence d'une croyance idiosyncrasique, telle que «Alfred croit que les fantômes existent », qui ne permet aucunement de statuer quant à l'existence effective des fantômes, les croyances «sociosyncrasiques», telles que «les Catholiques croient que le diable existe», permettent de déduire que le diable existe dans les sociétés catholiques. Le contexte des sociétés catholiques étant bien plus large que le cerveau d'Alfred, les objets qui y sont enchâssés se voient bel et bien octroyés une forme d'existence. Car une fois qu'ils ont été pris en charge par une autorité en Nous - autorité que l'on appellera désormais, par commodité, «nostrologique»leur sens peut être achevé et dépassé dans la référence ${ }^{26}$. En d'autres termes, le

24 D. Dennett, La stratégie de l'interprète. Le sens commun et l' univers quotidien, Paris, Gallimard, 1990, notamment pp. 229-266.

25 Les expressions entre guillemets sont de Descombes (1996), p. 79. Selon Descombes, l'ensemble des significations à la fois intentionnelles et impersonnelles forme un espace commun de référence bien réel, celui des usages établis. Cet espace commun assure la convergence des visées intentionnelles, car il est «ce à propos de quoi» les membres d'une société donnée sont susceptibles de parler, de penser ou de croire.

26 Ce néologisme «nostrologique», qui vise à désigner le Nous par analogie à la validation «égologique» du $J e$, est un peu barbare mais très parlant; il a été proposé de manière informelle par 
mode collectif des co-référenciations qui engendrent des objets intentionnels communs est synonyme «d'ontologisation».

L'identification et la détermination collectives, au XVIII ${ }^{\mathrm{e}}$ siècle, de l'objet notionnel «opinion publique» illustrent de manière remarquable cette transmutation statutaire ${ }^{27}$. Comme l'indique fort bien la métaphore de «l'élévation » du référent qu'utilise Francis Jacques, l' «opinion publique» a suivi une ascension sémantique qui l'a imposée comme une référence incontournable du contexte élargi que représente l'espace public - un contexte de plus en plus élargi puisqu'il part du cercle restreint de la sphère littéraire, s'étend au milieu plus englobant de la sphère judiciaire et atteint finalement l'espace décisif du politique. Grâce à ces élargissements contextuels qui constituent autant d'étapes clés de sa rigidification, l'entité possible qu'est l'«opinion publique»s'est vu peu à peu remplacée par une entité réalisée, sinon réelle, qui se voit ainsi élever à la qualité de référent. Bien que son «histoire transformationnelle» l'inscrive dans l'horizon d'une enquête politique dont elle n'est que la réponse provisoire, elle s'est détachée de son contexte d'origine pour se présenter comme le fondement nécessaire et immuable de la res publica moderne. Si l'identification terminale à laquelle a abouti le dialogue référentiel au long cours dont l'opinion publique a fait l'objet est reliée aux croyances premières de ceux qui ont œuvré à sa détermination, elle ne saurait donc aucunement s'y réduire. Au contraire, dans la mesure où l'opinion publique est «l'interréférence» du Nous logiquement indécomposable qui est à son principe, elle a conquis une plus-value en matière d'objectivité dont témoignent l'inertie et l'autonomisation de son réseau sémantique. Elle bénéficie en effet des propriétés d'extériorité et d'inaccessibilité dont sont généralement dotées les institutions sociales, que celles-ci renvoient aux valeurs républicaines, aux règles morales ou aux divinités mythologiques. Même si l'opinion publique n'est, du point de vue ontologique, que le corrélat intentionnel d'un Nous qui constitue le point de repère endogène et relativement invariant d'un collectif d'individus, elle s'impose donc comme si elle avait le statut exogène d'un état de choses.

Dans le cadre de l'analyse dialogique, la rétro-référenciation qui permet aux interlocuteurs de se rapporter simultanément à leur situation commune d'interaction et la co-référenciation à un état de choses sont les deux manifestations essentielles de la réalité auto-transcendante du Nous. D'une part, le phénomène de rétro-référenciation donne au Nous le pouvoir d'accomplir cela même qu'il exprime, à savoir l'existence d'un «individu collectif» qui se donne publiquement à reconnaître comme tel. Le Nous bénéficie ainsi du statut irréaliste mais «réalisant» d'un collectif nominal qui se construit par l'intermédiaire des actions

Kevin Mulligan dans plusieurs de ses interventions orales. D'un point de vue ontologique, il a l'avantage d'expliciter la teneur collective de l'instance de validation qui permet de créer des significations nouvelles et de fonder, à terme, des institutions.

27 Les exemples pertinents sont bien entendu innombrables. Par exemple, l'analyse que fait Oleg Kharkhordin de l'invention moderne de l'Etat pourrait très bien se prêter à une interprétation en termes de co-référenciation. En effet, il montre comment l'Etat a peu à peu conquis le statut d'un sujet d'action extrêmement puissant, d'une entité quasi mystique que personne ne voit mais dont chacun présume l'existence. O.Kharkhordin «What is the state? The Russian concept of Gosudarstvo in the European context », History and Theory 40, 2001, pp. 206-240. 
de co-référenciation dont il se fait le sujet. C'est d'ailleurs bien le statut saugrenu, tout à la fois nominal et réel, de la collectivité que la politique démocratique rend explicite: l'illusion unitaire du nouvel acteur historique que constituerait la société, même si elle n'est au départ que le produit des imaginations métaphysiquement enfiévrées, devient peu à peu une réalité effective. Comme le dit Jean Widmer, cette réalité, totalisante pour les individus empiriques et individuante pour leur groupe d'appartenance, représente une sorte de version collective du «cogito ergo sum [...]: le cogito suppose et ainsi fait exister l' ego» ${ }^{28}$. D'autre part, le phénomène de co-référenciation, qui est la deuxième manifestation de l'autotranscendance du Nous, préside à l'existence pesante d'un «intermonde» dont les allocutaires deviennent, bon an mal an, les obligés. En effet, le registre allocutif, chargé au départ des adhérences subjectives qui marquent l'interlocution, vacille lorsqu'il est placé sous l'égide d'un Nous élargi et stabilisé: il recule peu à peu devant le registre délocutif des objets intentionnels qui se sont autonomisés, tout au moins en apparence, des procédures de co-référenciation à leur origine. L'autonomisation de ces objets intentionnels, en supprimant le lien explicite qui les rattachait à l'autorité du Nous encore relativement localisable des interlocutions, les consacre comme des référents publics et impersonnels. C'est sous cet angle que des «inexistants» sociaux et politiques, tels l'opinion publique ou l'Etat, conquièrent le statut d'un référent «inter-mondain» dont la fixité relative est à même de provoquer et de contraindre en retour les visées intentionnelles dont pourtant, ontologiquement, ils dérivent.

\section{LA PRÉDICATION NOSTROLOGIQUE}

En vertu de la métaphysique politique qui permet tout à la fois aux individus de s'auto-constituer comme des agents collectifs et de constituer un univers sémantique et institutionnel intelligible, le Nous des allocutaires et le monde social émergent donc ensemble. Le constat de cette co-émergence, toutefois, n'implique aucunement l'adoption d'une conception intersubjective de la collectivité ou d'une version flottante des institutions publiques. En effet, bien que les espèces sociales et politiques restent ontologiquement dépendantes des croyances et des pratiques qui les reconduisent, leur épistémologie, c'est-à-dire leur connaissance, se trouve radicalement modifiée par la «référentialisation» de leurs contenus sémantiques ${ }^{29}$. Comme le dit John Searle, du point de vue de leur mode d'appréhension et non de leur mode d'existence, elles s'imposent aux membres de la communauté comme étant des vérités et des réalités objectives ${ }^{30}$. Cette étrange «objectivité-pour-nous», loin d'être une hallucination irraisonnée, est la conséquence logique des mécanismes d'auto-transcendance que nous avons esquis-

28 J. Widmer, «Langues et configurations de l'espace public » Hermès, 19, 1996, pp. 225-239

29 Nous privilégions ici l'acceptation anglo-saxonne de «l'épistémologie», qui renvoie à l'étude générale des modes possibles de la connaissance, de la logique et des caractéristiques des différents types de savoir existants. Nous ne renvoyons pas à son acceptation française qui désigne, quant à elle, la réflexion plus spécifique sur l'histoire, les méthodes et les résultats des sciences.

30 J. Searle, The Construction of Social Reality, Allen Lane, The Penguin Press, 1995. 
sés ${ }^{31}$. Tout comme le Nous peut s'imposer en retour sur ses constituants, les objets de pensée a priori imaginaires peuvent objectivement contraindre les esprits qui les ont engendrés. Or, le pouvoir contraignant dont bénéficie cette «objectivitépour-nous » est précisément l'enjeu même du politique: une fois, en effet, que l'on considère la co-référenciation comme le principe instituant par excellence, l'objet essentiel du politique devient le monde commun de référence qui donne sens aux expériences individuelles et aux actions collectives.

On comprend mieux, dans un tel contexte, la teneur fondamentalement discursive de la raison politique. Non seulement cette dernière stabilise, sélectionne, impose et hiérarchise les co-référenciations, mais elle les réifie dans des institutions qui peuvent exercer un pouvoir causal sur les actions individuelles, notamment via le système légal. À cet égard, les innovations linguistiques systématiques que tentent d'imposer les révolutionnaires de 1789 apparaît d'une grande lucidité sociologique. En prétendant transformer les choses et les individus en modifiant les mots qui les font advenir au langage, ils reconnaissent explicitement la langue comme étant la «méta-institution» dont l'appropriation est nécessaire à l'exercice du pouvoir ${ }^{32}$. La furie linguistique des révolutionnaires qui s'attaquent, comme le dit Jacques Guilhaumou, «non seulement aux choses que les mots représentent, mais aussi et surtout aux mots en personne», prend ainsi acte du pouvoir métaphysique de la langue politique ${ }^{33}$. La prise de conscience de ce pouvoir métaphysique, encore renforcée par le mythe révolutionnaire d'une scène sociale primitive qui aurait réussi à faire table rase du passé, confie à la «langue bien faite» des patriotes la lourde tâche de reconstruire «mot à mot» la nation. Ce sont les cérémonies de nomination politiques et les baptêmes juridiques qui sont chargés de faire advenir à l'existence cela même qu'ils dénomment. Car l'édification de la nouvelle République, prise dans l'urgence d'un événement qui se veut fondateur, ne dispose que du temps court de l'action publique: dans un tel contexte inaugural, ce que l'on pourrait appeler la prédication politique se voit lesté d'un poids sans commune mesure.

On peut privilégier ici les deux acceptions principales du terme de prédication. D'une part, il renvoie au sermon politico-religieux qui exhorte les fidèles citoyens à se conformer aux valeurs suprêmes qui sont censées gouverner leurs esprits. D'autre part, il renvoie à la qualification linguistique des événements, cette dernière affirmant la subordination des entités ou des sujets auxquelles il est fait référence (par ex., le roi) à un acte de volonté qui leur attribue des propriétés (par ex., l'acte de prédication «le roi est bon») ${ }^{34}$. Ainsi, dans la célèbre formule «l'opinion publique gouverne le monde», l'opinion publique est le sujet, alors que "gouverne le monde » manifeste la manière dont le sujet en question est décrit et pensé.

L'expression est d'Hilary Putnam, Raison, vérité et histoire, Paris, Minuit, 1984, pp. 66-68.

32 Sur le langage comme «méta-institution», voir Louis Quéré, «L'herméneutique constitue-t-elle un paradigme pour la sociologie?», Problèmes d'épistémologie en sciences sociales, t. 1, Paris, EHESS-CNRS, 1984.

33 Seule la citation est de J. Guilhaumou, La langue politique et la Révolution française. De l'événement à la raison linguistique, Paris, Méridiens Klincksieck, 1989, p. 15.

34 Pour une présentation très claire de ces notions, cf. P.F. Strawson, Les individus, Paris, Seuil, 1973. 
Mais par-delà son acception, laudative ou non, la prédication politique consiste de toute manière à imposer des connexions significatives à certains fragments particuliers de la réalité. Ce faisant, elle les subordonne à un ordre de sens plus général qui manifeste et affirme la puissance intentionnelle de ses énonciateurs. Cette notion de prédication, si on l'applique au niveau collectif auquel l'approche dialogique nous a permis d'accéder pour ainsi dire par «en-dessous », renvoie à l'ensemble des significations qui décrivent le monde sous un certain point de vue. Ce point de vue, tout à la fois intentionnel et impersonnel puisqu'il émerge dans «l'entre-nous» de la collectivité, est proprement politique: comme le dit Lefort, il instaure la description légitime du monde social ${ }^{35}$. L'espace politique apparaît, du coup, comme étant le lieu de la compétition entre les différentes «prédications originelles» auxquelles se livre le Nous ${ }^{36}$. Les prédications nostrologiques sont originelles dans la mesure où elles ne renvoient pas à des entités situées dans le monde empirique: on l'a vu, elles créent par co-référenciation des objets intentionnels - que ce soient des concepts, des institutions ou des lois. Toutefois, par rapport aux autres co-référenciations, les prédications politiques ont une propriété particulière qui leur confère une pesanteur sans pareille: le Nous qui est à leur principe n'est pas circonscrit à un espace social particulier car il est censé être valide pour toute la communauté.

\section{LE CONFLIT DES INTERPRÉTATIONS}

Dans le vaste contexte nominal que déploie l'ensemble des prédications possibles du monde social, la méta-description qui parviendra à s'imposer comme étant la seule description correcte de «ce qu'il y a» est un enjeu capital ${ }^{37}$. Car la méta-description politique régule et hiérarchise la multiplicité des activités et des biens en leur imposant, comme le dit Vincent Descombes, «un ordre de subordination $»$ de facture «architectonique» ${ }^{38}$. Elle prescrit les obligations des individus envers la cité et subordonne les activités et biens sociaux hétérogènes à la généralité supérieure et ultime qu'incarne la raison publique. C'est dire si la compétition qui oppose les différentes descriptions candidates à l'assentiment généralisé dont

35 Lefort (1978) op. cit.

36 Sur les prédications originelles, voir Oswald Ducrot, Les mots du discours, Paris, Minuit, 1980, pp. 57-92. Les prédications originelles sont différentes des prédications secondes, qui se contentent de rappeler les descriptions antérieures déjà accomplies dans le passé (par exemple, «ce discours a été très critiqué»); en effet, les prédications originelles effectuent ou se présentent comme si elles effectuaient des attributions nouvelles qui sont constitutives hic et nunc de la réalité qu'elles qualifient ( «j'ai trouvé que son discours était très orienté »).

37 Compte tenu de l'ontologie subjective et de la dimension artefactuelle qui caractérisent les faits sociaux et politiques, nous utilisons pour l'instant les notions de description, d'interprétation et de prédication politiques comme étant équivalentes entre elles. En effet, elles renvoient toutes à la définition-détermination du monde social: dans la mesure où il n'existe pas de réalité sociale et politique indépendante des significations que lui confère une communauté donnée, la distinction, tout au moins a priori, entre la description de «ce qui est» et l'interprétation de ce que pourrait signifier «ce qui est» n'a pas de sens.

38 V. Descombes, «Philosophie du jugement politique», La Pensée politique, ${ }^{\circ}$ 2, Seuil-Gallimard, 1994, pp. 131-157. 
bénéficie in fine la méta-description politique ne ressemble guère à un dialogue coopératif et pacifique. Elle renvoie à la lutte sans merci que se livrent les prédications concurrentes pour s'imposer comme étant le seul et unique système de référenciation socio-politique. La description victorieuse, une fois consacrée comme l'instance ultime qui est habilitée à départager le réel et l'imaginaire, le bien et mal, le juste et l'injuste, peut traiter en toute légitimité ses rivales comme des témoignages déficients, des opinions subjectives ou des idéologies partisanes.

Cette tentative de monopolisation de la bonne «version» du monde social est d'ailleurs loin d'être l'apanage des sociétés non démocratiques; bien au contraire, même dans les démocraties politiques où le conflit des interprétations du monde social est érigé en principe de gouvernement, les versions trop discordantes sont systématiquement évincées de la scène publique. Bien qu'une telle éviction prenne souvent la forme abusive d'une «police discursive» qui tente de discipliner la production des significations au profit du pouvoir en place, elle n'en est pas moins une nécessité politique ${ }^{39}$. En effet, les interprétations du monde social ne peuvent tourner en des versions radicalement disjonctives sans menacer la convergence minimale des différents points de vue individuels, nécessaire à la reconduction de la société. La «guerre des versions» du monde social qui caractérise l'espace politique - que ces versions prennent la forme encore floue des opinions dissonantes, la forme plus affirmée des courants d'opposition ou, plus récemment, la forme rigide des partis - ne peut être viable que si elle préserve le postulat selon lequel il existe bel et bien un monde social. Pour sauvegarder l'«unanimité de principe» qui garantit, par delà les divergences et les conflits situés, la commensurabilité des interprétations du monde social, une méta-description doit donc gagner du terrain sur ses concurrentes ${ }^{40}$. En confirmant la présomption d'un monde objectif, aussi nécessaire à l'équilibre architectonique de la société qu'à l'équilibre mental de ses membres, elle évite que le sens du monde social ne soit suspendu au conflit radical des interprétations - un conflit synonyme de désagrégation sociale. Grâce à une telle méta-description, l'organisation de la vie en commun ne sombre pas, comme le dit Foucault, dans «l'abîme d'un choix radical » qui laisserait libre cours au jeu hasardeux et arbitraire des dominations ${ }^{41}$. Seule une méta-description politique, en faisant office «d'agent herméneutique centralisateur», est à même de s'opposer à un cynisme incivique qui se baserait uniquement sur la loi du plus fort pour faire passer les valeurs et les règles du hasard à la nécessitée ${ }^{42}$.

Dans le cadre nominaliste de la politique, la méta-description qui permet de désamorcer l'imprévisibilité et l'inconstance du lien social et d'imposer les repères du juste et de l'injuste, du bien et du mal, est l'idéologie. Au sein d'une

M. Foucault, L'ordre du discours, Paris, Gallimard, 1971.

40 M. Pollner, Mundane Reason, Cambridge University Press, Cambridge, 1987. Cette unanimité principielle évite que le conflit des interprétations ne prenne la forme radicale d'une «guerre des mondes» qui mettrait en jeu, comme le suggère Nelson Goodman, des réalités totalement incompatibles. N. Goodman, Manières de faire des mondes, Nîmes, Ed. Jacqueline Chambon, 1992.

41 Foucault, « Nietzsche, la généalogie, l'histoire», in Hommage à Jean Hyppolite, Paris, PUF, 1971, pp. $145-172$.

42 Cette formule est de Olivier Cayla, «Droit», in M. Canto-Sperber (dir.) Dictionnaire d'éthique et de philosophie morale, Paris, PUF, 1996, pp. 439-446. 
société auto-déterminée qui a remplacé, comme le dit Lefort, «la parole du pouvoir» par «le pouvoir de la parole», l'idéologie doit pallier l'absence des instances extra-sociales qui assuraient la reproduction des sociétés traditionnelles ${ }^{43}$. Dans une société où, en principe, plusieurs interprétations du monde social sont en lutte pour imposer leur propre version de ce qui est et de ce qui serait souhaitable, elle offre un système global et surtout explicite d'interprétation du monde. Dans cette acception non péjorative, l'idéologie ne renvoie pas à la manipulation mensongère des masses silencieuses. Elle manifeste la vulnérabilité et la fragilité d'une unanimité de principe qui ne peut plus reposer sur la reconduction relativement aveugle de la tradition et doit être garantie par un travail idéologique de «colmatage» de l'être-ensemble. Si l'on suit Lefort ou Castoriadis, dans une société qui serait devenue lucide quant au mode de production endogène de ses institutions, l'idéologie doit conjurer le morcellement de la société en la resignifiant et en la «retotalisant»par un discours politique venu «d'en haut». En d'autres termes, c'est à l'idéologie qu'incombe le rôle de «centre de gravité narratif» que tenaient autrefois les mythes, la parole de Dieu ou le monarque ${ }^{44}$. Conformément à sa fonction «architectonique », elle doit hiérarchiser les priorités collectives et restreindre l'éventail des actions et des prédications possibles. En vertu de ce centre de gravité symbolique, la consécration de la société comme étant désormais le seul cadre ontologique de l'existence collective devient logiquement et politiquement viable. En effet, l'idéologie figure, bon an mal an, le point fixe qui permet à la collectivité de se percevoir comme une totalité unitaire: elle exprime l'autorité endogène du Nous qui résulte, on l'a vu, du procès d'autotranscendance propre aux co-référenciations. Reste à savoir si le Nous ainsi exprimé par la méta-description idéologique répond bel et bien à une configuration dialogique ou si, au contraire, il se caractérise par une organisation hiérarchique dont le modèle dialogique ne suffit pas à rendre compte.

\section{L'EXPERTISE POLITIQUE DES SIGNIFICATIONS PUBLIQUES}

La perspective dialogique nous a permis d'inscrire dans une société d'allocutaires historiquement située les matériaux nominaux de la construction de leur «intermonde» social et politique. Cela étant, une telle perspective, en misant sur la coopération équilibrée et symétrique des libres participants à un Nous préoccupé, qui plus est, de vérité et de rationalité, tend à une forme d'idéalisation sociologiquement peu crédible. Car appréhendé d'un point de vue plus sociologique, un tel équilibre coopératif est une chimère: la disparité des conditions sociales, politiques et économiques, ainsi que le système de compétences qui leur est intrinsèquement rattaché, le rendent pratiquement impossible. Sans remettre en question les contributions de l'approche dialogique à notre ontologie du politique, il

43 Lefort (1980) p. 349

44 Sur cette notion de centre de gravité narratif, qu'il utilise quant à lui à l'échelle de la conscience individuelle, voir D.Dennett, «Comment nous tissons notre moi», in H. Grivois et J.-P. Dupuy (dir.) Mécanismes mentaux, mécanismes sociaux. De la psychose à la panique, Paris, La Découverte, 1995, pp. 147-166. 
nous faut donc tenter de caractériser quelle peut bien être la teneur du «déséquilibre» coopératif qui caractérise la détermination de la «chose» publique - car déséquilibre il y a, y compris dans les dialogues référentiels.

Contrairement en effet à ce que postule le mirage coopératiste de l'approche dialogique, tous les individus n'ont pas la même maîtrise linguistique et conceptuelle des significations en usage et ils ne disposent pas du même accès aux entités auxquelles ces significations sont censées référer. Ainsi, la plupart des espèces naturelles sont identifiables par des critères que seuls les experts scientifiques sont capables d'appliquer, conformément à ce qu'Hilary Putnam appelle «la division sociale du travail linguistique ${ }^{45}$. Des concepts apparemment anodins, tel «l'eau», ont des propriétés empiriques qui ne peuvent être certifiées que par les paramètres objectifs dont les experts ont la maîtrise - en l'occurrence, la composition moléculaire H2O. Les agents ordinaires, tout en partageant les compétences linguistiques que requiert le cours de la vie quotidienne, délèguent ainsi aux experts la détermination des référents qui leur sont matériellement et cognitivement inaccessibles. Cette délégation, le plus souvent consentie, reconnaît l'autorité de ceux qui ont les moyens de déterminer rigidement les référents des concepts scientifiques dont l'usage commun, en revanche, demeure flou et indécis: seuls les forestiers, par exemple, sont à même de différencier l' «hêtre » d'un « orme » ${ }^{46}$. Si l'on suit Putnam, les membres ordinaires de la communauté prennent donc acte de la répartition sociale des compétences et reconduisent sans sourciller des significations instituées dont le travail de validation leur échappe.

Il reste alors à préciser si cette répartition des compétences, qui autorise et contraint tout à la fois les membres de la communauté à s'en remettre aux spécialistes, touche également les concepts sociaux et politiques. A l'encontre de l'idéalisation dialogique, il semble bien que la division du travail linguistique se retrouve également dans la forme d'expertise que constitue la connaissance ordinaire du monde social. Or, une telle division, relativement bénigne pour les espèces naturelles, a des conséquences drastiques sur la fixation conceptuelle et la rigidification linguistique des significations publiques qui meublent l'intermonde social. En effet, dans la mesure où de telles significations déterminent les orientations politiques et structurent le champ de l'expérience collective, tous les membres de la communauté devraient en principe participer à leur fixation. Mais une telle exigence, non seulement démocratique mais grammaticale puisque le Nous de société englobe par définition tous les $J e$ qui le composent, paraît bien compromise par la hiérarchie sociale de la connaissance. Elle paraît d'autant plus compromise qu'elle change de statut lorsqu'elle se déplace du domaine réservé de la science au domaine des faits institutionnels : elle passe de l'exercice légitime de l'expertise, justifié par l'autorité de la compétence, à l'autorité tout court des privilégiés de l'ordre social et politique.

Lorsqu'elle touche les concepts sociaux et politiques, la teneur essentiellement épistémologique de la division du travail linguistique dont traite Putnam devient un problème fondamentalement politique: celui de la division du travail social. Les concepts sociaux et politiques, une fois appréhendés dans le cadre

45 Sur la «division du travail linguistique », cf. Hilary Putnam, «The meaning of meaning», in Mind, Langage and Reality, Cambridge, Cambridge University Press, 1975, pp. 215-271.

46 Putnam (1975) op. cit. 
d'une «coopération» non seulement structurée mais intéressée, ne peuvent être considérés comme les produits d'une collaboration rationnelle. Ils doivent être rapatriés sous l'égide des intérêts particuliers de tous ceux qui profitent des avantages discursifs et cognitifs de leur position pour orienter les significations en fonction de leur propre vision du monde. Ce détournement intéressé est d'autant plus aisé que la teneur exclusivement intentionnelle des concepts sociaux et politiques les prive de toute confrontation avec des entités matérielles. Contrairement aux concepts scientifiques, en effet, de tels concepts ne renvoient pas à des «particuliers de base », c'est-à-dire à des entités observables dont les propriétés spatiotemporelles contraignent la «référence identifiante» dont elles font l'objet ${ }^{47}$. Les objets notionnels ne pouvant opposer le même type de résistance que les objets matériels aux discours qui les désignent, ils sont totalement perméables aux attributions orientées que leur imposent les prédicateurs politiques. L'autorité politique, si tant est qu'on l'appréhende, avec Keith Baker, comme une «autorité discursive » qui statue sur les interrelations des acteurs sociaux, a donc toute latitude pour exploiter cette perméabilité à son avantage ${ }^{48}$.

En d'autres termes, bien que tous les membres de la collectivité soient en droit de participer à la détermination de «l'objectivité-pour-nous» de leur intermonde, de facto, peu d'entre eux sont conviés à être les véritables experts de la «chose» publique. Même dans une démocratie où la seule parole publique légitime est la parole proférée au nom du public, les mandataires du peuple ont les moyens d'imposer leur propre définition du monde social. Ces moyens, loin de relever du mauvais esprit et des ambitions occasionnelles de ceux qui sont parvenus à s'approprier la volonté générale à leur propre compte, sont pour ainsi dire structurels. Ils sont procurés par l'instance suprême chargée de gérer les prédications originelles du Nous de société: l'Etat. En effet, du point de vue ontologique comme du point de vue normatif, l'Etat est un objet intentionnel démesuré que les co-référenciations collectives, ainsi que leurs objectivations dans des dispositifs institutionnels légaux, militaires et policiers, sont parvenues à stabiliser. En monopolisant l'exercice de la violence légitime au nom de l'arbitraire suprême qu'est la raison d'Etat, il arrête la sélection des candidats illimités à la convention sociale. Autrement dit, l'Etat, qui est par définition l'émanation de haut-niveau du Nous de société, bénéficie de tous les avantages de l'auto-transcendance. Tout en restant formellement tributaire de la puissance intentionnelle du Peuple en tant qu'entité fictive, idéelle dont il est tout à la fois l'incarnation et la représentation, il s'autonomise du peuple concret et lui impose en toute légitimité ses décrets. Une fois scellée par «la bouche artificielle du droit», «la souveraineté nominale» de l'Etat, même si elle n'est ontologiquement qu'une construction conventionnelle, tend à prendre la forme d'une «tyrannie légale ${ }^{49}$. L'Etat, devenu candidat hors jeu dans la guerre des versions qui opposent les candidats au pouvoir, est à même de remplir la fonc-

47 Sur la notion de «référence identifiante», réservée aux «particuliers » de base, c'est-à-dire aux entités spatio-temporelles publiquement observables, comme les choses et les personnes, cf. Strawson (1973) op. cit.

48 K.M. Baker, Au tribunal de l'opinion. Essais sur l'imaginaire politique au XVIII' siècle, Paris, Payot, 1993, p. 14.

49 L'Etat prend cette forme dans sa version absolutiste comme dans sa version démocratique. A ce propos, cf. Cayla (1996) p. 336. 
tion herméneutique ultime que constitue l'imposition de la bonne interprétation du monde social. Car l'autorité devenue tierce de l'Etat lui permet de court-circuiter la nature allocutive et dialogique des référents collectifs et de leur conférer le statut délocutif des «vérités » publiques que chacun est tenu de connaître.

Les professionnels de la prédication politique que sont les hommes d'Etat, tout en prétendant porter la parole du Peuple, s'improvisent ainsi les porteurs d'une vérité qu'ils seraient seuls habilités à proférer. Ces "porteurs de vérité», une fois consacrés ou auto-consacrés comme les délégués du Peuple, sont les nouveaux piliers de l'étrange métaphysique politique qui permet de convertir les «mots» de quelques-uns en des «choses» pour la plupart. Ils deviennent, peu ou prou, les «méta-experts» de l'objectivité socio-linguistique qui procure aux membres d'une communauté leurs termes de référence. Les «experts» politiques, en s'intronisant les délégués à l'élaboration des référents et des normes communautaires, ont toute latitude pour revêtir leurs intérêts particuliers de la légitimité de l'intérêt général. C'est précisément à la croisée de cette traduction fallacieuse des intérêts particuliers en un intérêt général qu'intervient le travail minutieux de l'idéologie. Une idéologie comprise, cette fois, non dans le sens neutre et intégratif de la métadescription chargée de suppléer à l'absence des religions et des mythologies, mais dans le sens péjoratif d'une instance manipulatrice au service de la domination.

\section{L'IDÉOLOGIE OU LA POLITIQUE DE LA VÉRITÉ}

La méta-description politique, on l'a vu, a un statut nostrologique: elle est l'émanation, provisoire et relativement stabilisée, du conflit des interprétations qui divisent et rassemblent tout à la fois les membres de la collectivité. Mais lorsqu'elle occulte sa dimension prédicative de point de vue pour s'imposer comme un discours légiférant qui paraît n'être le discours de personne, qui est émis de nulle part et qui s'offre, par là même, à tous, elle se fait purement et simplement idéologie $^{50}$. Car, paradoxalement, le vrai gagnant du conflit politique des interprétations est celui qui réussit à occulter son statut de prédicateur. En présentant son point de vue comme étant le témoignage objectif et naturalisant de la vérité intrinsèque des événements, il évite une mise en perspective subjective qui dégage, par définition, de multiples possibilités de contestation. Or, les prédications idéologiques, en occultant leur position située pour se prétendre les porte-voix de l'universel, transgressent la nature argumentative du langage politique. En effet, le principe de la politique repose, comme le dit Hannah Arendt, sur la doxa, sur l'opinion, bref sur que les hommes peuvent changer au gré de leur seule volonté, et non sur l'épistémé, la science ou la vérité ${ }^{51}$. Alors que la démocratie suppose que tous les citoyens ont la possibilité d'atteindre une doxa correcte et que personne ne possède une épistémé des choses politiques, l'idéologie prétend produire une véritable science du politique ${ }^{52}$. La vérité bénéficie d'une infaillibilité de prin-

\footnotetext{
Lefort (1978) p. 251

H. Arendt, La crise de la culture, Paris, Gallimard, 1972, notamment pp. 305-336.

52 Castoriadis (1990) p. 285.
} 
cipe qui est nécessairement tyrannique, car elle est précisément ce qu'il n'est pas possible de changer, fût-ce au terme d'un échange argumenté. Lorsque la politique est revêtue des oripeaux fondamentalement apolitiques de la vérité, le dissident tend à être traité comme celui qui ose prétendre que $2+2=5$, c'est-à-dire comme un ignorant à rééduquer, un fou à soigner ou un idiot à écarter ${ }^{53}$.

La politique de la vérité qui régit l'idéologie dans sa version «distordante» revient donc à dénaturer l'action politique, qui est une action essentiellement nominale à laquelle sont en principe conviés tous les participants au Nous collectif. Une fois la description légitime du monde social monopolisée par les métaexperts des significations publiques, le peuple, par définition inexpert, se voit interdit toute possibilité d'intervention politique. Car les experts disposent d'un avantage déterminant par rapport à la masse «impensante» et laborieuse que constitue la «démocrassouille ${ }^{54}$. Ils disposent d'emblée, comme le dit Bourdieu, de «la position de survol» propre au point de vue général - qui est aussi celui « du général»-de ceux qui sont «mandatés pour produire un point de vue qui est reconnu comme transcendant par rapport aux points de vue singuliers $(. ..) »^{55}$. Les maîtres des vérités publiques, au nom de leur prétendue expertise, s'approprient ainsi à leur propre compte ce que Michaël Bakhtine appelle «l'activité mentale du nous»; ils s'intronisent les «architectes» d'un édifice idéologique dont les membres ordinaires ne sont plus que les «locataires $»^{56}$. Les opinions particulières des prédicateurs idéologiques, une fois transformées en une vérité pour tous par une universalisation forcée, ne peuvent plus tolérer les interprétations divergentes. L'expertise de la prétendue essence des institutions est désormais réservée aux «Maîtres de la signification» que sont les Idéologues (hommes d'Etat, journalistes, juges, enseignants, etc) - des maîtres qui trônent, si l'on suit Castoriadis, bien au-dessus du «Maître de la violence légitime » ${ }^{57}$. Les idéologues, en présentant leur prédication particulière comme étant l'expression même de la Vérité et de l'intérêt général, imposent la description enchantée, esthétisante, bref, faussement désintéressée, d'un monde social qui serait enfin débarrassé du conflit objectif des intérêts. En annexant peu ou prou la structure temporelle des événements historiques et l'imprévisibilité des libertés particulières à une prédication supérieure, ils expriment le fantasme de la toute-puissance nominale d'un pouvoir qui rêve de créer, sous le seul effet de la politique, une réalité commune. La perte du principe de réalité et le rétablissement d'une «société sans histoire » au sein même

53 Joseph Gabel, La fausse conscience. Essai sur la réification, Paris, Minuit, 1962.

54 Sur l'analyse des processus anti-démocratiques inhérents à la démocratie, cf. G. Duprat, «Introduction », in G. Duprat (dir.) L'ignorance du peuple. Essais sur la démocratie, Paris, PUF, 1998, p. 1-16.

55 P. Bourdieu, La distinction, Paris, Minuit, 1979, p. 520, P. Bourdieu, Choses dites, Paris, Minuit, 1987, p. 148.

56 Mikhail Bakthine, Le marxisme et la philosophie du langage, Paris, Minuit, 1977, p. 31.

57 Castoriadis, Le monde morcelé. Les carrefours du labyrinthe III, Paris, Seuil, 1990, p. 123. Rappelons ici que le programme originel des Idéologues du $18^{\mathrm{ème}} \mathrm{sc}$. consistait précisément à développer une science naturelle et rationaliste des idées, visant à transformer les comportements humains en un domaine d'expertise. Une telle science des idées se donnait pour objectif la constitution, la rigidification et l'expertise des pensées. Cf A.Destutt de Tracy, Eléments d'idéologie, Paris, Vrin, 1979. 
de la société historique est donc le prix à payer de la méta-description idéologique: «Meurs et deviens une institution», voilà la malédiction qu'elle impose à la réalité individuelle et collective ${ }^{58}$.

L'aspiration à un tel pouvoir performatif, aussi absolu qu'instantané, est une forme tendanciellement pathologique de la prédication politique. Dans son acception «noble», en effet, la prédication politique, tout en hiérarchisant l'importance et la visibilité des différents aspects de la totalité sociale, reste orientée vers l'intérêt général et le bien public. Dans son acception intéressée et instrumentale, en revanche, elle n'est plus orientée vers le bien-être des citoyens; elle érige artificiellement et implicitement une des parties du Nous collectif en totalité de référence. Si l'on reprend ici les termes de Jon Elster, l'idéologie est donc bien «le tout compris selon la logique de la partie ${ }^{59}$. Elle constitue une prédication métonymique qui exploite la division du travail linguistique et social au profit d'une minorité d'experts. Ces experts, tout en se prétendant l'émanation du Nous de société et en dissimulant, par là même, leur statut de prédicateurs, exploitent le pouvoir ontologique de la collectivité au profit de leurs seuls intérêts particuliers. Leurs prédications, restreintes à un point de vue et à un contexte de description socialement et politiquement situés, usurpent «l'universel particulier» que constitue la communauté dans son ensemble. Et pourtant, de telles prédications, même si elles sacrifient au sociocentrisme d'un groupe de particuliers, peuvent continuer, grâce aux «lois de la réfraction idéologique», à se réclamer de «l'activité mentale du nous ${ }^{60}$. Tout en imposant leurs propres interprétations, intéressées et particulières, du monde social, elles parviennent à tisser les fils sémantiques qui servent de trame à toutes les relations sociales. C'est cette curieuse universalisation qui scelle la réussite suprême de l'idéologie.

\section{CONCLUSION}

Nous avons vu, tout au long de ce parcours, que le nominalisme qui accompagne l'avènement de la politique démocratique a libéré l'histoire et le monde social des entités occultes qui les tenaient prisonniers. Il a ainsi dévoilé au grand jour le secret des institutions: loin d'être le fruit du déploiement anhistorique d'une téléologie supérieure, elles sont sans essence ${ }^{61}$. Dans ce nouveau cadre, potentiellement émancipateur, la cohésion de la communauté repose désormais explicitement sur les accords dialogiques, les objets notionnels et les descriptions convergentes qui font du monde social un monde commun. La politique peut être ainsi décrite comme le procès explicite de co-référenciation par lequel le Nous de société détermine et hiérarchise les objets intentionnels communs qui lui permettront, en retour, d'exister comme une totalité supérieure aux parties qui la compo-

\footnotetext{
58 La première citation est de Lefort (1978) p. 296. La seconde de Bourdieu (1987) p. 193.

59 Jon Elster, «Un historien devant l'irrationnel. Lecture de Paul Veyne» Information sur les sciences sociales, $19,1980, \mathrm{n}^{\circ} 4-5$, p. 790

$60 \quad$ Bakhtine (1977) p. 34

61 Foucault (1971) «Nietzsche, la généalogie, l'histoire», p. 148.
} 
sent. Ce Nous, toutefois, n'a pas la configuration égalitaire et coopératiste qui caractérise les dialogues référentiels désintéressés de la vie ordinaire: il est structuré par une division interne du travail linguistique et social qui réserve à un petit nombre d'élus l'expertise et la détermination des significations publiques. L'appropriation de la prédication nostrologique, c'est-à-dire de la méta-description du monde social à laquelle tous les membres du Nous de société doivent consentir, devient alors l'enjeu politique par excellence. Les experts des méta-descriptions politiques que sont les idéologues tendent à transformer cette appropriation en monopolisation, notamment en dissimulant le statut prédicatif, et donc intentionnel et situé, de leurs discours sous la vérité prétendue de leurs décrets. Ces porteurs de vérité, en prétendant faire de leurs désirs une réalité et de leurs vertus une nécessité, manifestent l'imposture d'un Nous particulier, privé de la légitimité d'un Nous véritablement collectif.

On pourrait arguer que notre définition de $l a$ et $d u$ politique comme étant un procès collectif de co-référenciation, dûment chargé de maintenir l'unité minimale de la société et d'assurer la félicité publique, est par trop «descriptiviste» et langagière. En effet, leur caractérisation en termes d'interprétation et de co-référenciation semble faire peu de cas des actions, des institutions et des autorités qui les incarnent. Mais cette caractérisation quelque peu flottante est essentiellement due au cadre ontologique que nous avons adopté ici: ce dernier suspend par définition la question de la réalisation factuelle des prédications politiques. Bien évidemment, dans la société empirique, les «vérités » publiques et les credos idéologiques n'épuisent aucunement la raison politique: celle-ci prend corps dans les luttes de classe, les intérêts économiques et les actions partisanes comme dans la raison ordinaire des expériences vécues et des usages établis sans lesquels elle ne serait, littéralement, qu'une langue morte. La méta-description politique, tout en ayant ontologiquement la puissance intentionnelle de créer de toutes pièces un espace commun de référence, compose pratiquement avec les mœurs, les normes et les structures qui meublent d'ores et déjà le monde social. D'une certaine manière, il faudrait donc prendre au pied de la lettre la notion même de méta-description politique. Comme toute description, elle est la description de «quelque chose»- un «quelque chose» avec lequel elle doit imaginer des accommodements puisqu'il s'agit du système, devenu largement anté-prédicatif et donc implicite, des mœurs établies, des préjugés tacites et des pratiques routinières. Le sens en circulation dans les prédications politiques en quête de nouveaux référents publics n'est que le sommet, constamment renégocié, de «l'iceberg social» que constitue le sens provisoirement «congelé» de l'«objectivité-pour-Nous».

Notre approche ontologique du politique n'évite pas seulement, me semble-til, la critique de descriptivisme, mais aussi celle de réductionnisme et d'individualisme. En effet, l'existence des faits sociaux et politiques étant ramenée sous les auspices d'un Nous qui est non seulement indécomposable mais structuré, les institutions ne peuvent être réduites au résultat provisoire d'un contrat établi par une assemblée d'individus. Le Nous au principe des institutions, loin d'être un sujet collectif pluriel qui ne serait que l'agrégation des Je qui le composent, dépasse logiquement la somme de ses membres. En vertu de sa structure interne, de son auto-transcendance et de son objectivation dans des dispositifs publics, il constitue une instance politique concrète qui a ses propres lois et son propre 
pouvoir d'action. Ainsi, le Nous de la communauté, qu'il prenne la forme empirique de l'Etat-nation, de la majesté royale ou des porte-parole du Public, peut déclarer la guerre, signer un traité, refuser des accords commerciaux, défendre un idéal commun, etc. ${ }^{62}$ Soumis par définition à l'autorité de ce Nous, les particuliers n'ont pas les moyens cognitifs et politiques de lui opposer une interprétation alternative ou une expérience idiosyncrasique. Car contrairement à ce que suggère Searle, l'ontologie subjective des institutions n'est pas une ontologie à la première personne du singulier, mais à la première personne du pluriel. Loin d'être réductibles à l'autorité à la première personne des opinions, des actions ou des impressions individuelles, les institutions s'imposent comme des réalités factuelles sur lesquelles les actions individuelles sinon collectives n'ont plus prise ${ }^{63}$.

C'est dire si la première étape de notre enquête ontologique, qui a privilégié la face prédicative des faits sociaux et politiques, doit être suivie par une deuxième étape, qui constitue par ailleurs l'ordinaire de la démarche sociologique: celle qui consiste à rendre compte de la face obligeante des prédications nostrologiques. Les objets intentionnels communs que produisent ces dernières, une fois soutenus par des rapports de coopération, d'autorité et d'expertise, et réifiés dans des dispositifs institutionnels, acquièrent une consistance objective. Ainsi transformés en des faits institutionnels, ils se présentent à tous les membres de «l'entre-nous » comme étant dotés de l'apparence objective d'une chose en soi. A mon sens, cette étrange «chosification» constitue le cœur même de l'ontologie politique - une ontologie qui se propose de reprendre à nouveaux frais les fondements de la res publica $^{64}$.

Institute for Social Research

University of Michigan

426 Thompson str.

PO Box 1248

Ann Arbor, MI 48106

USA

\section{BIBLIOGRAPHIE}

Arendt, H. La crise de la culture, Paris, Gallimard, 1972.

Baker, K.M. Au tribunal de l'opinion. Essais sur l'imaginaire politique au XVIII' siècle, Paris, Payot, 1993.

Bakthine, M. Le marxisme et la philosophie du langage, Paris, Minuit, 1977.

62 V. Descombes, «A propos de la 'critique du sujet' et de la critique de cette critique», Cahiers Confrontation, $\mathrm{n}^{\circ}$ 20, 1989, pp. 115-129.

63 Pour les conséquences d'une telle approche sur l'analyse des phénomènes dits à la première personne, je me permets de renvoyer à mon article «Esprit, es-tu là? Le sociologue et l'autorité de la première personne», Information sur les Sciences Sociales/Social Science Information, vol.38, 1999, n $^{\circ}$ 2, pp. 203-248.

${ }^{64}$ La préparation de cet article a eu la chance de bénéficier du soutien financier du Fonds National de la Recherche Scientifique Suisse, dans le cadre du programme «Demain la Suisse», bourse $n^{\circ} 84$ SP-056189. 
Bourdieu, P. La distinction, Paris, Minuit, 1979.

Bourdieu, P. Choses dites, Paris, Minuit, 1987.

Castoriadis, C. Domaines de l' homme. Les carrefours du labyrinthe II, Paris, Seuil, 1986.

Castoriadis, C. Le monde morcelé. Les carrefours du labyrinthe III, Paris, Seuil, 1990.

Cayla, O. «Droit», in M. Canto-Sperber (dir.) Dictionnaire d'éthique et de philosophie morale, PUF, 1996, pp. 439-446.

Chisholm, R. «Intentionality», The Encyclopedia of Philosophia, New York, Macmillan, 1972.

Dennett, D. La stratégie de l'interprète. Le sens commun et l'univers quotidien, Paris, Gallimard, 1990.

Dennett, D. «Comment nous tissons notre moi » in H.Grivois et J-P Dupuy (dir.) Mécanismes mentaux, mécanismes sociaux. De la psychose à la panique, Paris, La Découverte, 1995, pp. 147-166.

Descombes, V. «A propos de la «critique du sujet» et de la critique de cette critique», Cahiers Confrontation, $\mathrm{n}^{\circ} 20,1989$, pp. 115-129.

Descombes, V. «Philosophie du jugement politique», La Pensée politique, $\mathrm{n}^{\circ}$ 2, Seuil-Gallimard, 1994, pp. 131-157.

Descombes, V. Les Institutions du sens, Paris, Minuit, 1996.

Destutt de Tracy, A. Eléments d'idéologie, Paris, Vrin, 1979.

Ducrot, O. Les mots du discours, Paris, Minuit, 1980.

Duprat, G. «Introduction», in G. Duprat (dir.) L'ignorance du peuple. Essais sur la démocratie, Paris, PUF, 1998, pp. 1-16.

Dupuy, J-P. Introduction aux sciences sociales. Logique des phénomènes collectifs, Paris, Ed. Marketing, 1992.

Elster, J. «Un historien devant l'irrationnel. Lecture de Paul Veyne», Information sur les sciences sociales, $19, \mathrm{n}^{\circ} 4 / 5,1980$, pp. 773-804.

Foucault, M. «Nietzsche, la généalogie, l'histoire», in Hommage à Jean Hyppolite, Paris, PUF, 1971, pp. 145-172.

Foucault, M. L'ordre du discours, Paris, Gallimard, 1971.

Gabel, J. La fausse conscience. Essai sur la réification, Paris, Minuit, 1962.

Goodman, N. Manières de faire des mondes, Nîmes, Ed. Jacqueline Chambon, 1992.

Guilhaumou, J. La langue politique et la Révolution française. De l'événement à la raison linguistique, Paris, Méridiens Klincksieck, 1989.

Hacking, I. The social construction of what?, Cambridge/London, Harvard University Press, 1999.

Jacques, F. Dialogiques I. Recherches logiques sur le dialogue, Paris, PUF, 1979.

Jacques, F. Différence et subjectivité. Anthropologie d' un point de vue relationnel, Paris, Aubier, 1982.

Jacques, F. Dialogiques II. L'espace logique de l'interlocution, Paris, PUF, 1985.

Kaufmann, L. «Esprit, es-tu là? Le sociologue et l'autorité de la première personne », Information sur les Sciences Sociales/Social Science Information, vol. 38, 1999, n² 2, pp. 203-248.

Kharkhordin, O. "What is the state? The Russian concept of Gosudarstvo in the European context», History and Theory 40, 2001, pp. 206-240.

Largeault, J. Enquête sur le nominalisme, Paris/Louvain, Nauwelaerts, 1971.

Latour, B. Petite réflexion sur le culte moderne des dieux faitiches, Paris, Ed. des Empêcheurs de tourner en rond, 1996.

Lefort, C. Les formes de l' histoire. Essais d'anthropologie politique, Paris, Gallimard, 1978.

Lefort, C. «Penser la révolution dans la Révolution française», Annales, n² 2, 1980, pp. 334-352.

Panaccio, C. Les mots, les concepts et les choses. La sémantique de Guillaume d'Occam et le nominalisme aujourd' hui, Montréal/Paris, Bellarmin/Vrin, 1991.

Pollner, M. Mundane Reason, Cambridge University Press, Cambridge, 1987.

Putnam, H. «The meaning of meaning in Mind, Langage and Reality, Cambridge, Cambridge University Press, 1975, pp. 215-271. 
Putnam, H. Raison, vérité et histoire, Paris, Minuit, 1984.

Quéré, L. «L'herméneutique constitue-t-elle un paradigme pour la sociologie?», Problèmes d'épistémologie en sciences sociales, t. 1, Paris, EHESS-CNRS, 1984.

Quine, W.V.O. Le mot et la chose, Paris, Flammarion, 1977.

Quine, W.V.O. «On What There Is» in S S. Laurence et C. Macdonald (ed.) Contemporary Readings in the Foundations of Metaphysics, Oxford, Blackwell, 1998, pp. 32-45.

Searle, J. L'Intentionalité. Essai de philosophie des états mentaux, Paris, Minuit, 1985.

Searle, J. «Intentionalistic Explanations in the Social Sciences», Philosophy of the Social Sciences, vol. 21, 1991, n³, pp. 331-344.

Searle, J. The Construction of Social Reality, Allen Lane, The Penguin Press, 1995.

Strawson, P.F. Les individus, Paris, Seuil, 1973.

Van Inwagen, P. «The Nature of Metaphysics» in S. Laurence \& C. Macdonald (éd.) Contemporary Readings in the Foundations of Metaphysics, Oxford/Massachusetts, Blackwell, 1998, pp. 11-21.

Widmer, J. «Langues et configurations de l'espace public», Hermès, 19, 1996, pp. 225-239. 